\title{
Coordinated Multipoint: Concepts, Performance, and Field Trial Results
}

\author{
Ralf Irmer, Vodafone \\ Heinz Droste, Deutsche Telekom \\ Patrick Marsch, Michael Grieger, and Gerhard Fettweis, Technische Universität Dresden \\ Stefan Brueck, Qualcomm CDMA Technologies GmbH \\ Hans-Peter Mayer, Alcatel-Lucent Bell Labs \\ Lars Thiele and Volker Jungnickel, Fraunhofer Heinrich-Hertz-Institut
}

\begin{abstract}
Coordinated multipoint or cooperative MIMO is one of the promising concepts to improve cell edge user data rate and spectral efficiency beyond what is possible with MIMOOFDM in the first versions of LTE or WiMAX. Interference can be exploited or mitigated by cooperation between sectors or different sites. Significant gains can be shown for both the uplink and downlink. A range of technical challenges were identified and partially addressed, such as backhaul traffic, synchronization and feedback design. This article also shows the principal feasibility of COMP in two field testbeds with multiple sites and different backhaul solutions between the sites. These activities have been carried out by a powerful consortium consisting of universities, chip manufacturers, equipment vendors, and network operators.
\end{abstract}

\section{INTRODUCTION}

High spectral efficiency (i.e., high aggregated cell data rate per unit of spectrum) is especially important for data networks. Mobile data traffic has recently surged due to the availability of affordable data dongles, notebooks, tablet computers with third-generation $(3 G)$ radio modules, and smartphones with web-oriented user interfaces. Vodafone, for example, has observed 70 percent growth of data traffic within one year for their European mobile networks. So far, $3 \mathrm{G}$ networks could support the traffic growth. However, eventually, more efficient wireless technology and novel deployment concepts like small cells and heterogeneous networks are needed to provide the required capacity.

Ubiquitous user experience is key for the end user to have a guaranteed minimum service quality corresponding to a minimum data rate. Denser network deployments address this issue caused by low link budget at the cell edge. However, this goes along with larger areas where the transmission is limited by interference.

Long Term Evolution (LTE) and mobile WiMAX use multiple-input multiple-output (MIMO)-orthogonal frequency-division multiplexing (OFDM) and achieve improved spectral efficiency within one cell. However, inter-cell interference is still preventing these technologies from coming close to the theoretical rates for multi-cell networks. There are two fundamental ways to deal with inter-cell interference: Coordination of base stations to avoid interference and constructive exploitation of interference through coherent base station cooperation. Conceptually, we extend single-cell MIMO techniques, such as multi-user (MU-MIMO), to multiple cells.

This article shows results from the EASY $-\mathrm{C}^{1}$ project, which focused on coordinated multipoint (COMP) from 2007 to 2010 and set up two multisite testbeds for LTE-based COMP in Dresden and Berlin. ARTIST4G ${ }^{2}$ and other forthcoming projects will continue to use these platforms.

COMP is a main element on the LTE roadmap beyond Release 9. In LTE Release 11, some simpler COMP concepts may appear, but it is generally expected that advanced COMP concepts will take longer to be mature enough for commercial use.

The main scope of this article is to outline the basic COMP concepts, and highlight the potentials and technical challenges when introducing them in future mobile networks. Moreover, we sketch practical COMP schemes for uplink and downlink, assess their performance in large-scale network simulations, and use field trials in urban areas to demonstrate the maturity of COMP. 


\section{COORDINATION AND COOPERATION in Mobile NetWorks}

One key element of mobile radio networks is spatial reuse (i.e., the reuse of resource elements such as timeslots or frequency bands) in a geographical distance, where the signal strength is reduced due to path loss, shadowing, and so on. Historically, this was achieved using network planning with certain frequency reuse patterns, which have, however, the drawback of poor resource utilization. $3 \mathrm{G}$ and $4 \mathrm{G}$ technologies are using full frequency reuse, which in turn leads to interference between the cells.

In $[1,2]$ network coordination has been presented as an approach to mitigate intercell interference and hence improve spectral efficiency. Figure 1 shows the cooperation architecture for COMP. The same spectrum resources are used in all sectors, leading to interference for terminals (user equipment [UE] in Third Generation Partnership Project [3GPP] terminology) at the edge between the cells, where signals from multiple base stations are received with similar signal power in the downlink. Multiple sectors of one base station (eNB in 3GPP LTE terminology) can cooperate in intrasite COMP, whereas intersite COMP involves multiple eNBs.

The sectors at one site can be different selfsustained units, or different remote radio heads linked via fiber to a central baseband unit. The eNBs may be interconnected by the logical X2 interface. Physically, this could be a direct fast fiber link, or a multi-hop connection involving different backhaul technologies.

The cooperation techniques aim to avoid or exploit interference in order to improve the celledge and average data rates. COMP can be applied both in the uplink and downlink. All schemes come with the cost of increased demand on backhaul (high capacity and low latency), higher complexity, increased synchronization requirements, more channel estimation effort, more overhead, and so on. The aim of this article is to highlight the potentials of COMP and its technical challenges to be addressed for introducing it in next-generation mobile networks.

\section{Evaluation by SimULATION AND FIELD TRIALS}

Different approaches to COMP can be analyzed using system-level simulations with hexagonal cells and evaluation methodologies customary in the 3GPP, Next Generation Mobile Networks (NGMN), and International Telecommunication Union (ITU). Unless otherwise specified, the intersite distance in all computer simulations has been set to $500 \mathrm{~m}$, a terminal speed of 3 $\mathrm{km} / \mathrm{h}$ is assumed, and the system bandwidth is $10 \mathrm{MHz}$.

The results of such simulations will be presented in this article. However, it is not enough to evaluate the feasibility of an approach solely based on simulations. Field trials are essential to find out the critical technical issues, and they encourage an end-to-end view. The EASY-C

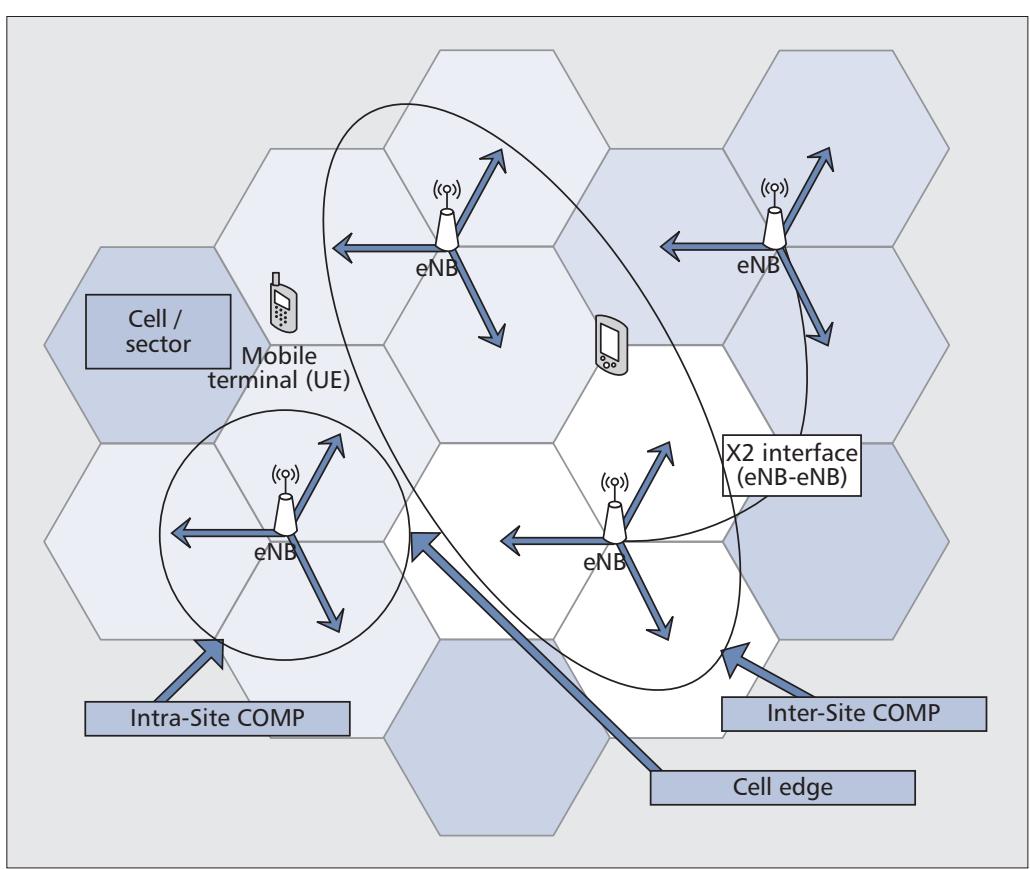

Figure 1. Base station cooperation: intersite and intrasite COMP.

project has set up two outdoor testbeds with slightly different underlying technology and focus, as shown in Table 1; see also [3-5].

\section{UPLINK COORDINATED MULTIPOINT}

\section{OVERVIEW}

Theoretical work has shown that uplink (UL) COMP offers the potential to increase throughput significantly $[1,2]$, in particular at the cell edge, which leads to enhanced fairness overall. Modeling some practical aspects such as a reasonably constrained backhaul infrastructure and imperfect channel knowledge, UL COMP promises average cell throughput gains on the order of 80 percent, and roughly a threefold cell edge throughput improvement [6]. The channel information is available in the network without resource-consuming feedback transmissions in the uplink. Also, the terminals need no modifications in order to support UL COMP. Therefore, base station cooperation may be easier to implement than in the downlink (DL). Only the interface between base station sites (X2) needs to be defined. In case of joint detection in the UL, higher X2 capacity is needed than for joint transmission in the DL. Although the UL capacity is not the bottleneck in today's networks, guaranteeing a minimum data rate, especially for cell edge users, is improving user experience, and UL COMP may be used to carry control traffic necessary to implement DL COMP.

In general, the UL COMP schemes can be classified as:

Interference-aware detection: Here, no cooperation between base stations is necessary; instead, base stations also estimate the links to interfering terminals and take spatially colored interference into account when calculating receive filters (interference rejection combining).

Joint multicell scheduling, interference pre- 


\begin{tabular}{|c|c|c|}
\hline Environment & \multicolumn{2}{|c|}{ Dense urban } \\
\hline Trial setup & 10 sites with up to a total of 28 sectors & 4 sites with up to 10 sectors \\
\hline Frequency & \multicolumn{2}{|c|}{$2.68 \mathrm{GHz} \mathrm{DL}, 2.53 \mathrm{GHz} \mathrm{UL}$} \\
\hline Baseline technology & $\begin{array}{l}\text { OFDMA in DL and UL, scalable bandwidth } 5-20 \\
\mathrm{MHz} \text {, transmissions limited to a maximum of } 40 \\
\text { resource blocks (PRBs) in UL and } 10 \text { PRBs in DL. }\end{array}$ & $\begin{array}{l}\text { DL: } 2 \times 2 \text { MIMO-OFDMA, UL: } 1 \times 2 \text { SC-FDMA, } \\
\text { scalable bandwidth } 1.5-20 \mathrm{MHz} \text {, full bandwidth } \\
\text { can be used in both up- and downlink }\end{array}$ \\
\hline Processing & $\begin{array}{l}\text { Real-time DL transmission. For uplink COMP } \\
\text { offline processing. Scheduling is investigated in } \\
\text { quasi-realtime. }\end{array}$ & $\begin{array}{l}\text { Real-time PHY, adaptive MIMO multiple access and } \\
\text { network layer. PHY is extended for DL CoMP. }\end{array}$ \\
\hline Backhaul and interconnects & $\begin{array}{l}\text { 5.4/5.8 GHz microwave with a net data rate of } \\
100 \mathrm{Mb} / \mathrm{s} \text { and } 1 \mathrm{~ms} \text { delay }\end{array}$ & $\begin{array}{l}1 \mathrm{~Gb} / \mathrm{s} \text { Ethernet over optical fiber and free-space- } \\
\text { optical links. }\end{array}$ \\
\hline Testbed scope & $\begin{array}{l}\text { UL and DL MU-MIMO COMP, relaying, practical } \\
\text { issues }\end{array}$ & $\begin{array}{l}\text { DL MU-MIMO, COMP, relaying, real-time demos } \\
\text { such as high-definition mobile video conference }\end{array}$ \\
\hline
\end{tabular}

Table 1. COMP testbeds developed within the EASY-C project.

diction, or multicell link adaptation, requiring the exchange of channel information and/or scheduling decisions over the X2 interface between base stations [7].

Joint multicell signal processing. Here, degrees of freedom exist in the way that decoding of terminals may take place in a decentralized or centralized way, and to which extent received signals are preprocessed before information exchange among base stations. In general, there is a trade-off between using backhaul efficiently by a maximum extent of preprocessing (e.g., as in distributed interference subtraction, DIS, where decoded data is exchanged), but obtaining less CoMP gain, or using a large backhaul capacity (as in the case of the distributed antenna system, DAS, where quantized receive signals are exchanged) and obtaining a better performance.

\section{Selected Simulation Results}

In the following section selected UL cooperation schemes are introduced. During performance evaluation it is distinguished between gains of intrasite and intersite cooperation, where intersite cooperation needs X2 backhaul capacity.

Uplink Interference Prediction - The basic idea of UL interference prediction [7] is to perform link adaptation based on predicted signalto-interference-plus-noise ratio (SINR) values that are likely to occur during the associated data transmissions. Prediction is enabled by exchange of resource allocation information within a cluster of cooperating cells. In addition, the UL receivers provide channel state information related not only to their associated terminals, but also to the strongest terminals of neighboring cells. Due to interference prediction, more appropriate link adaptation can be realized, and hence the performance can be improved. The exchange of resource allocation information between two cells causes only moderate backhaul traffic in the range of $8 \mathrm{Mb} / \mathrm{s}$. Whereas performance gains with intrasite coop- eration prove to be rather low, we observe up to 25 percent gain in spectral efficiency and 29 percent gain with respect to baseline cell edge throughput if intersite cooperation including up to six interfering cells is simulated. The prediction accuracy degrades if the channel state information gets outdated. Therefore, the X2 latency should not exceed $1 \mathrm{~ms}$, even at low terminal speed.

Uplink Joint Detection - Uplink joint detection means that signals received at different sectors are jointly processed [8]. Hence, virtual MIMO antenna arrays may spread out over different users as well as different base station sectors at the network side. Most of the information exchange between cooperating cells is caused by sharing the quantized baseband samples received in each cell. Channel state information and resource allocation tables are shared in the cooperation cluster as well. First estimates reveal that even with consideration of less than half the cooperation cluster size as described above for interference prediction, the cell-to-cell X2 traffic would exceed $300 \mathrm{Mb} / \mathrm{s}$ for $10 \mathrm{MHz}$ system bandwidth. This high amount of backhaul traffic motivates the investigation of intrasite joint detection. In case of intersite joint detection including up to three sectors per terminal, gains in spectral efficiency and cell edge throughput account for 35 and 52 percent, respectively (2). Sticking with intra-site joint detection, the improvements drop only moderately to 25 percent on average and 24 percent at the cell edge (3).

Combining high throughput and low latency as required by joint detection will cause a cost burden for the backhaul, specifically the X2 interface. Therefore, a combination of intrasite joint detection (no X2 needed) and intersite interference predictions (low throughput demand) has been considered. This even outperforms the throughput-demanding intersite joint detection, as shown in Fig. 2. However, the burden of low-latency $\mathrm{X} 2$ remains. 

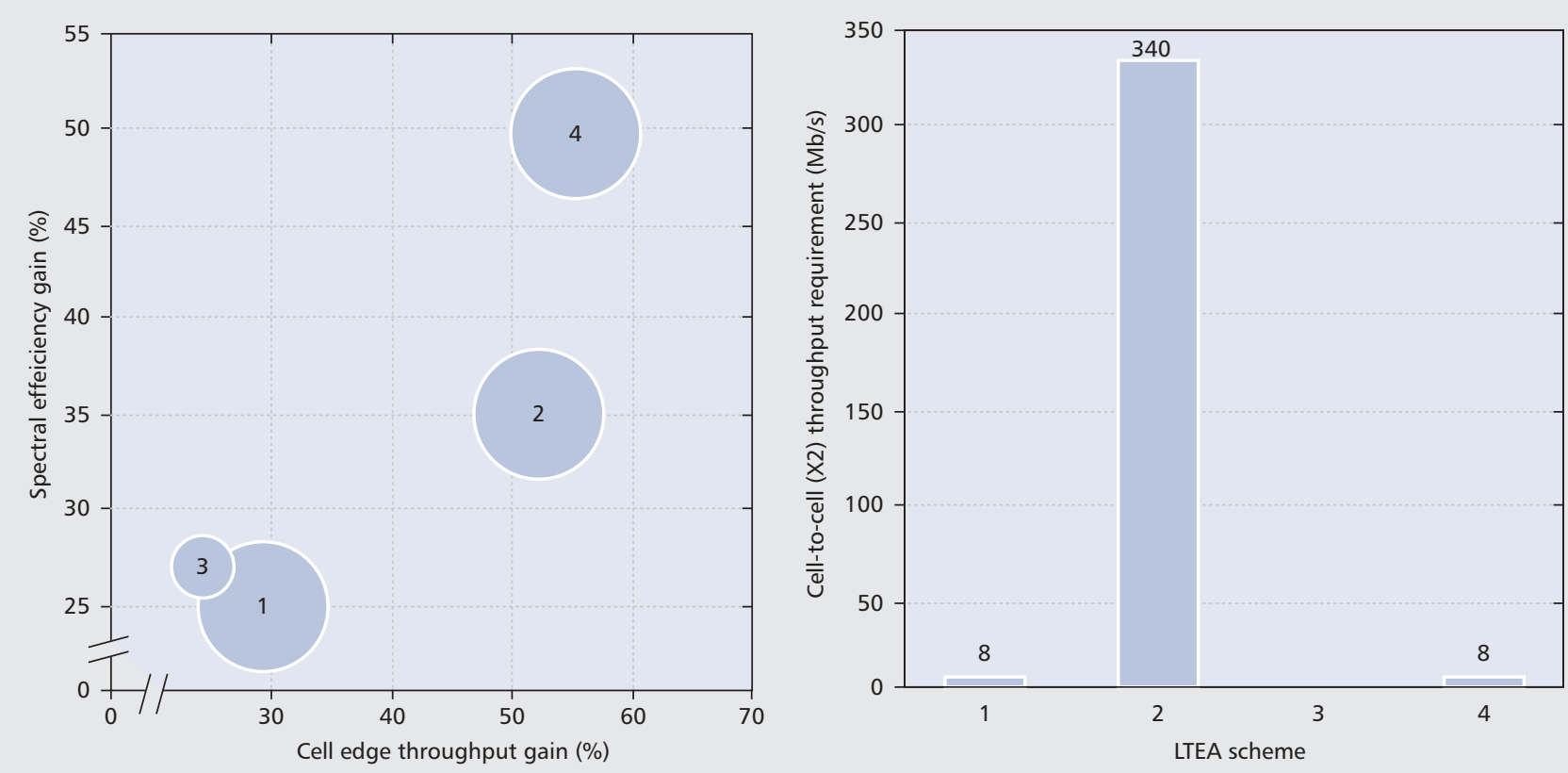

Figure 2. Performance of selected uplink COMP schemes: 1) inter-site interference prediction, 2) inter-site joint detection, 3) intra-site joint detection, 4) combining inter-site interference prediction with intra-site joint detection.

\section{Selected Field Trial Results}

Joint decentralized and centralized detection of terminals was evaluated in the Dresden testbed [9]. Two terminals with one transmit antenna each transmitted continuous sequences of modulation and coding schemes, which were received by two base stations with one receive antenna each (KATHREIN 80010541). The scenario resembled a symmetric cell edge scenario, but the terminals were moved such that interference conditions changed continuously. The receive signals were recorded so that different cooperation schemes could be applied and evaluated offline.

The result plot in Fig. 3 shows the average rates that could be achieved with different cooperation strategies vs. the backhaul required. Here, square and round markers are used to distinguish both UE types. We can see that in an LTE Release 8 system, where each UE unit is decoded only by the serving base station, an average rate of about $1.5 \mathrm{~b} / \mathrm{channel}$ use is possible for UE 1 (square marker). This can be improved to about $2.2 \mathrm{~b} /$ channel use simply if a flexible (i.e., transmission time interval [TTI]wise) assignment of UE to eNBs is enabled, with the option of local decoding with successive interference cancellation (SIC). A further rate improvement of UE 1 is possible if DIS is enabled, where one UE unit is decoded first at one eNB, and the decoded data are then forwarded to the other eNBs for interference subtraction, requiring a smaller extent of backhaul. This scheme turns out to reduce the outage probability significantly. The remaining points show the performance of a DAS, where the eNBs exchange quantized received signals, with either 6 or 12 bits per antenna both for I and Q signal dimensions. As compared to LTE Release 8 , in this scenario full DAS-based cooperation can improve the average throughput by about 70

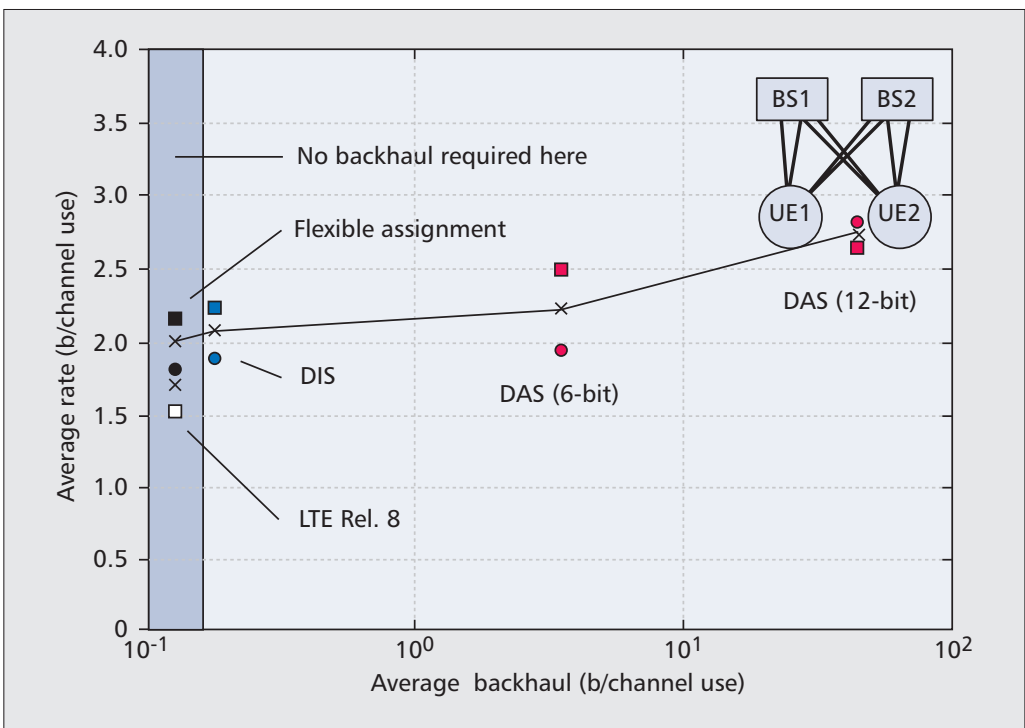

Figure 3. Achieved rates vs. required backhaul for different uplink cooperation schemes, as measured in field trials.

percent, but the backhaul required is more than two orders of magnitude larger than for decentralized concepts (DIS). Further measurements have shown that DIS schemes become even more valuable in asymmetric scenarios, such that an adaptive usage of centralized and decentralized cooperation schemes depending on the interference situation appears promising.

The presented results provide evidence of the potential benefits of using CoMP in specific scenarios. Figure 4 shows the COMP gains in a large-scale setup in the EASY-C testbed in downtown Dresden with 12 eNBs on five sites. The spacing between the sites is $350-600 \mathrm{~m}$, with an antenna height of 15-35 m. Two UE units are 


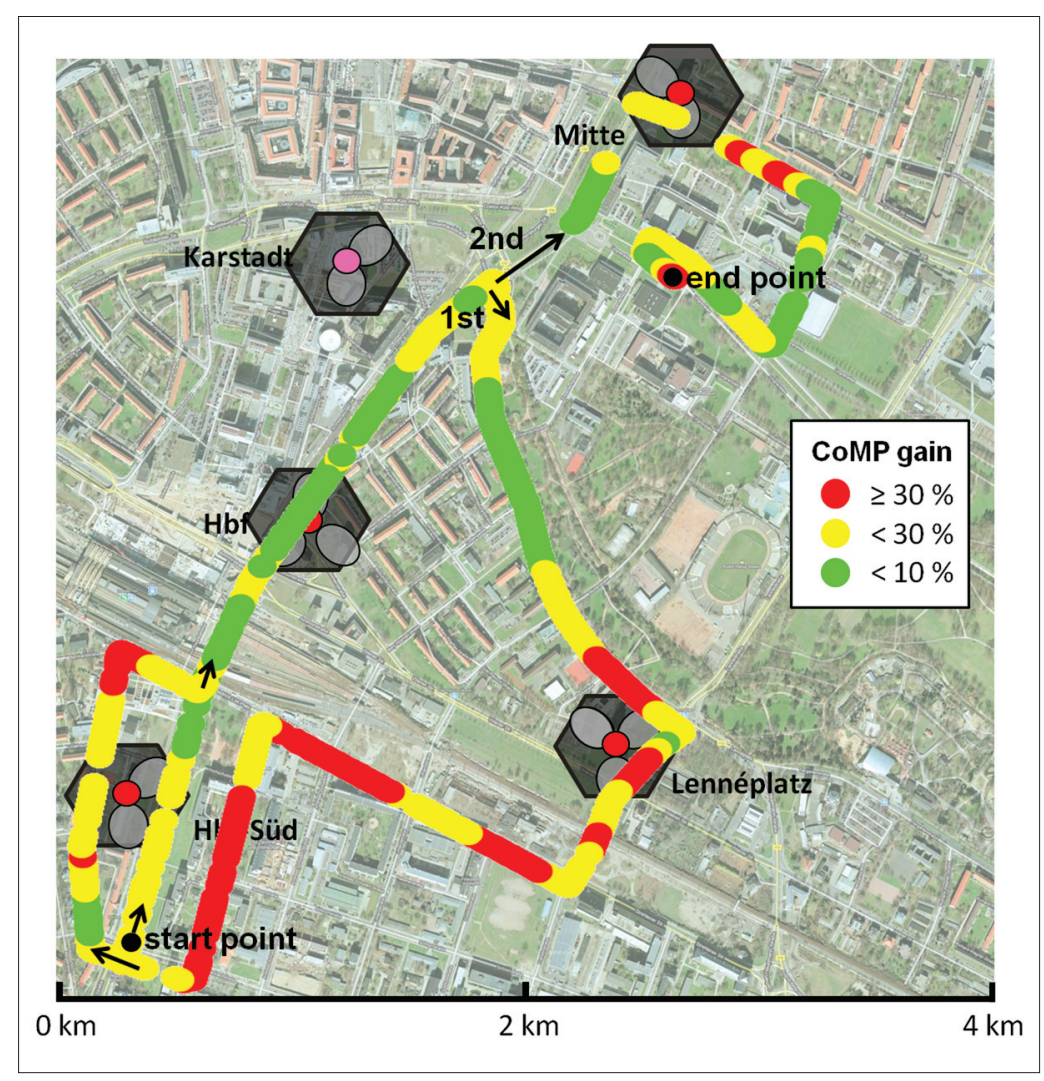

Figure 4. Uplink COMP gains in EASY-C testbed in downtown Dresden.

carried on a measurement bus on a $7.5 \mathrm{~km}$ length route, as depicted in Fig. 4, which passes through different kinds of surroundings, including an underpass, apartment buildings, a train station, and open spaces like parking areas. Conventional non-cooperative decoding is compared to cooperative joint decoding. Through cooperation, average spectral efficiency gains of about 20 percent were achieved. In certain areas, however, gains above 100 percent were observed. Furthermore, the variance of achievable rates at different UE positions was reduced, corresponding to fairer rate distribution throughout the measurement area.

\section{Challenges}

From the experience of implementing and testing UL COMP, the following key challenges have become apparent.

Clustering: Suitable clusters of cooperating base stations have to be found, which can be done in a static way or dynamically, as discussed below.

Synchronization: Cooperating base stations have to be synchronized in frequency such that intercarrier interference is avoided, and in time in order to avoid both intersymbol and intercarrier interference [10]. The maximum distance of cooperating base stations is limited since different propagation delays of different terminals may conflict with the guard interval. This aspect may be compensated through a more complex equalization.

Channel estimation: A large number of eNBs in the COMP cluster in the UL will require a larger number of orthogonal UL pilot sequences.
At some cluster sizes, the COMP gains are outweighed by capacity losses due to additional pilot effort.

Complexity: The above mentioned field trials have been performed using orthogonal frequency-division multiple access (OFDMA) in the UL, as this enables a subcarrier and symbol-wise MIMO equalization and detection in the frequency domain. If single-carrier (SC)-FDMA was used as in LTE Release 8, equalization would be more complex.

Backhaul: It can be a severe issue if centralized decoding is applied. Hence, adaptive decentralized/centralized cooperation appears to be an interesting option. Furthermore, source coding schemes appear interesting for backhaul compression.

\section{DOWNLINK CoORdinAted MULTIPOINT}

\section{OVERVIEW}

Base station cooperation in the DL can also improve average throughput and, more important, cell edge throughput [2]. 3GPP distinguishes between the following categories of DL COMP [11].

Coordinated scheduling/beamforming: User data is only available in one sector, the so-called serving cell, but user scheduling and beamforming decisions are made with coordination among the sectors.

Joint processing COMP: User data to be transmitted to one terminal is available in multiple sectors of the network. A subclass of joint processing is joint transmission, where the data channel to one terminal is simultaneously transmitted from multiple sectors.

Both coordinated scheduling/beamforming and joint transmission have been investigated within the EASY-C project.

\section{Selected Simulation Results}

Coordinated beam selection [12] and coscheduling are part of the investigated COMP schemes. Co-scheduling draws its gains from interference avoidance and is less complex than DL joint transmission. One approach which includes beamforming per cell is presented here. Synchronization of the cells is needed; however, there is no strict requirement on phase stability as known from coherent techniques. Multicell co-coordinated beamforming has been assessed in system-level simulations taking into account the latency for inter-NodeB communication.

The method is based on an extended precoding matrix index (PMI): the terminals measure and report the PMIs for their own cells (best companion) and additionally the PMIs for the neighboring cells causing the strongest interference (worst companion) plus the channel quality information for the case that these worst interferers are not used.

The multicell scheduler is based on a distributed approach, with overlapping clusters of seven neighboring cells each. The scheduling is coordinated within the clusters. The following results are given for four closely spaced antennas 
at the base station and two UE antennas at 20 $\mathrm{MHz}$ system bandwidth.

The simulations show significant gains for coordinated DL scheduling, in particular for mobiles at the cell edge. Additionally, the gains were evaluated for different radio channels and different latencies for communication between the sites. As can be observed in Fig. 5, $1 \mathrm{~ms}$ latency/hop has only a moderate impact on the gains. Even with highly timevariant channels such as urban macrocell (UMa) at $30 \mathrm{~km} / \mathrm{h}$, co-scheduling still provides a sensible improvement. Assuming $6 \mathrm{~ms}$ latency per hop, the gains are still preserved for UE velocities up to $3 \mathrm{~km} / \mathrm{h}$.

The aggregated additional traffic on the backhaul sums up to approximately $5 \mathrm{Mb} / \mathrm{s}$ for 20 $\mathrm{MHz}$ spectrum; as a result this technique is also economically attractive.

\section{Selected Field Trial Results}

Since joint transmission is regarded as the most challenging CoMP technique from the implementation perspective, it has been implemented in both testbeds to investigate the feasibility of coherent transmission for intra- and intersite COMP [13, 14]. Significant throughput gains have been demonstrated for specific interference scenarios. The same techniques have also been assessed in wide-area system-level simulations to study more complex scenarios. The following enabling features were essential for the trials:

- Sufficient timing and frequency synchronization accuracy: In the trials GPS was used, although network-based approaches such as IEEE 1588v2 could also provide sufficient accuracy.

- Low-phase-noise radio frequency (RF) oscillators were used.

- Cell-specific reference signals.

- Time-stamped CSI feedback.

- Synchronous exchange of data and channel state information (CSI) between eNBs over the X2 interface.

- Distributed precoding and the provision of precoded pilots.

An example of the DL COMP experiments conducted in the Berlin testbed is shown in Fig. 7a. A distributed implementation of joint transmission has been demonstrated with synchronized base stations and cell-specific pilots. Terminals estimate the multicell channel and feed the CSI back to their serving cells. Base stations exchange CSI as well as data and independently perform pre-coding with the goal to maximize the desired signals whilst minimizing mutual interference.

Quantization and compression of the CSI are important topics, but outside the scope of this trial setup. CSI is fed back from the terminals safely using UL resources at a data rate of 4.6 $\mathrm{Mb} / \mathrm{s}$. Feedback interval and precoding delay are 10 and $20 \mathrm{~ms}$, respectively. The X2 interface between base stations is realized using a $1 \mathrm{~Gb} / \mathrm{s}$ Ethernet connection over cooper, fiber, or freespace optics, depending on the setup. The bidirectional load is $300 \mathrm{Mb} / \mathrm{s}$ realized with $0.5 \mathrm{~ms}$ latency.

Measurements were taken in the laboratory [5] and over the air in both indoor and outdoor

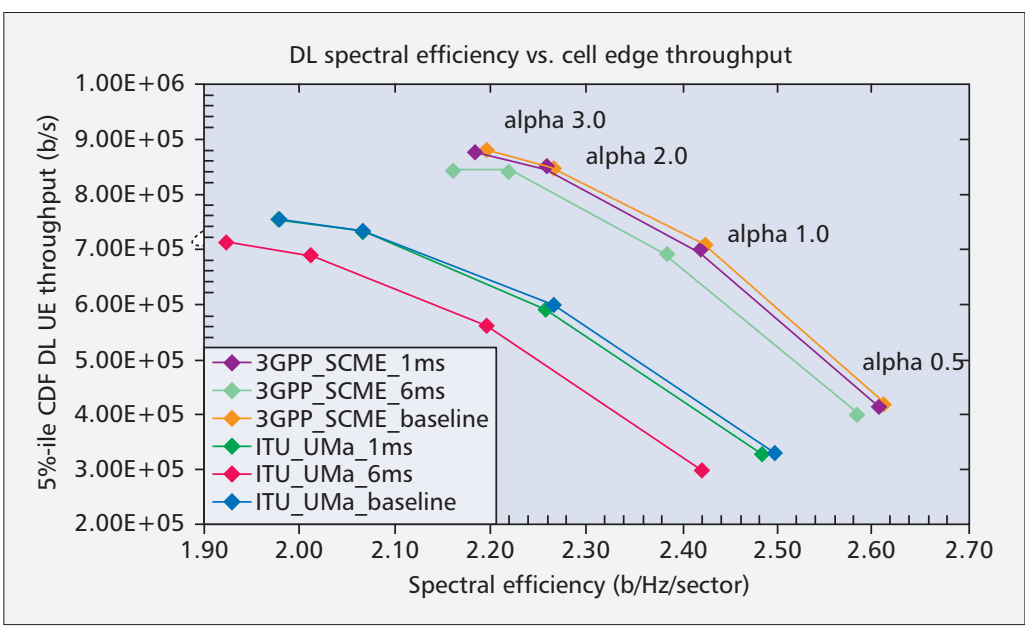

Figure 5. Downlink co-scheduling: spectral efficiency vs. cell edge throughput for ITU UMa and SCME radio channels and different backhaul latencies.

environments (Fig. 6, bottom left). It was observed that the interference situation experienced at a terminal is indeed critical at the cell edge if both base station signals are received equally strong on average at full frequency reuse. Signal and interference links fade independently, and sometimes the signal is stronger than the interference, while after a very short distance the opposite can be true. This is the origin of the high outage probabilities observed at the cell edge in the interference-limited case (Fig. 6, bottom right). Once DL COMP is switched on, significantly higher data rates can be realized in both cells simultaneously, due to the mutual interference cancellation. Moreover, the outage probability is remarkably reduced. Our experiments have shown that COMP gains are significant for simple interference scenarios, and that the implementation challenges can be overcome.

In reality, non-cooperating cells would surround the cluster of cooperative cells, leading to a remaining interference floor not yet present in our trials. The presence of such external interference has been studied in wide-area systemlevel simulations using basically the same COMP concept also tested in the field. Note that the active set is found so that in each cell two users are randomly placed, and each user gets only one stream. In all cells, only those user sets requesting the same cooperation cluster are investigated. In Fig. 6 (top right) we observe that there is no gain from using explicit CSI feedback in the serving cell, exploited for multi-user DL beamforming. Performance is equivalent to a fixed grid-of-beams as in LTE Release 8 if the terminal estimates in addition the surrounding interferers coherently, applies interference rejection combining, and provides implicit frequencyselective feedback on interference-aware PMI and CQI, and the base station applies scorebased scheduling [4]. Explicit CSI feedback is useful for CoMP. With increasing cluster size, the interference floor is reduced and the performance enhanced accordingly, at the cost of additional effort for overhead and backhaul. For more details, see [5]. 


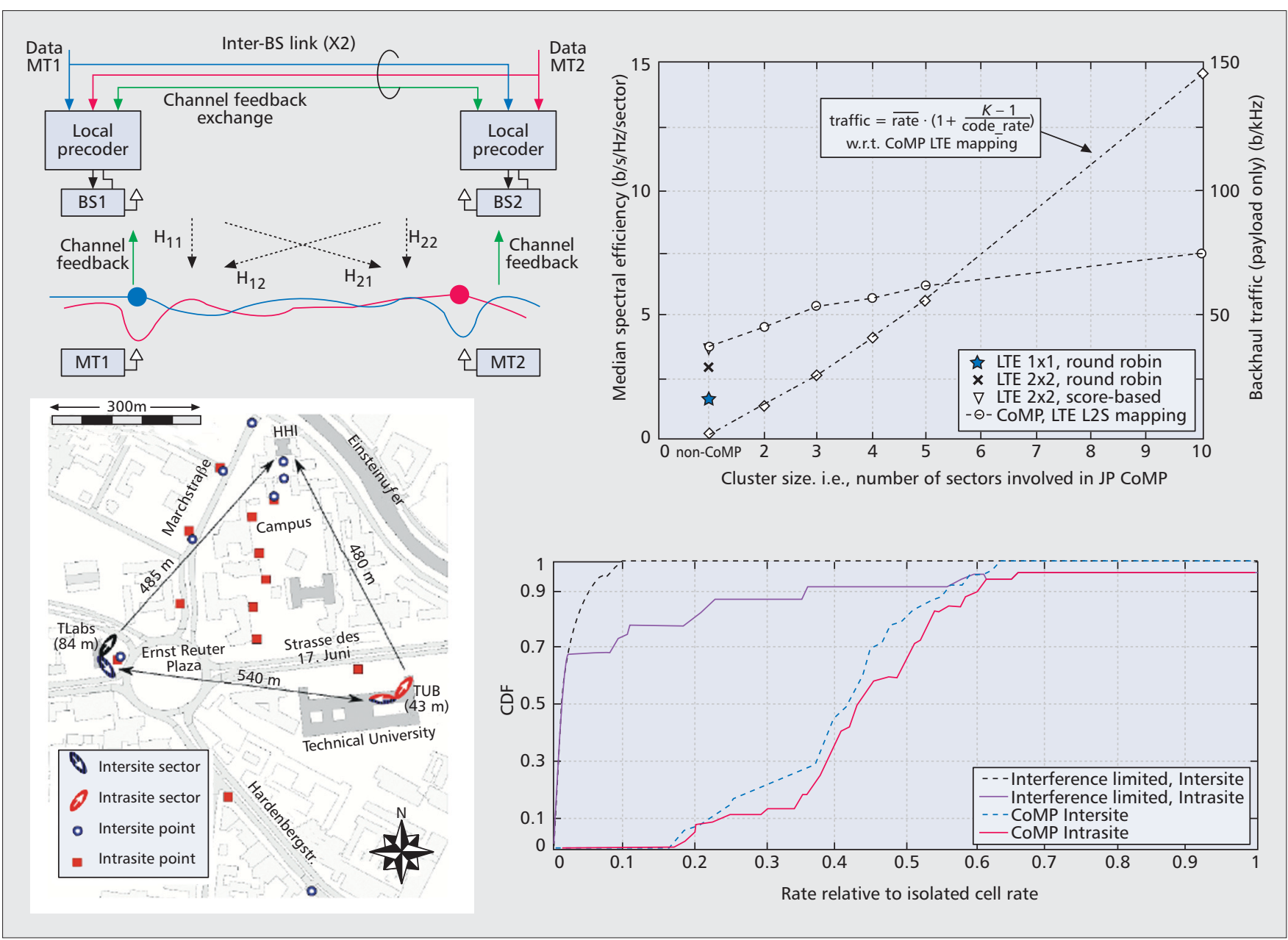

Figure 6. Top left: Distributed implementation of Joint transmission COMP. Top right: Performance and backhaul traffic vs. cluster size obtained from system-level simulations. Bottom left: Intra- and inter-site test scenarios in Berlin [5]. Bottom right: Measured throughput with full frequency reuse in a two-cell scenario w/o external interference relative to the case of isolated cells.

\section{Challenges}

Our results indicate that the complexity of DL COMP can be managed in real-world scenarios and that significant gain can be realized by forming small cooperation clusters in large-scale networks. However, solutions for the following are needed before it can be integrated in next-generation mobile networks:

- Reduced cost of base station synchronization and low-phase-noise transmitters

- Efficient feedback compression

- Reduced feedback delay

- Efficient channel prediction at the precoder

- Flexible formation of cooperation clusters

- Handling of outer interference within the cluster

- Efficient multi-user selection

- Flexible networking behind COMP

- Integration of COMP into higher layers

\section{Clustering of Cells}

As demonstrated in previous sections, COMP has the capability to enhance spectral efficiency and cell edge throughput significantly. However, COMP requires additional signaling overhead on the air interface and over the backhaul in case of intersite cooperation.
Therefore, in practice only a limited number of base stations can cooperate in order to keep the overhead manageable. The cooperating cell clusters should be set up adaptively based on RF channel measurements and UE positions in order to exploit the advantages of COMP efficiently at limited complexity. A key requirement for any adaptive cluster algorithm is that it fits into the architecture of the radio access and/or the core network of LTE. The 3GPP standard already offers a framework for selforganizing networks (SONs) to support automatic configuration and optimization of the network. Within EASY-C an adaptive mobilestation-aware clustering concept has been designed that can be integrated with small standard changes to the existing network architecture and the SON concept of LTE.

In order to evaluate the performance of the adaptive clustering concept, system-level simulations were run employing a hexagonal network layout shown in Fig. 7a. The scenario was configured with 193 -sector sites of $500 \mathrm{~m}$ intersite distance. The 3 GPP UMa spatial channel model (SCM) at $2 \mathrm{GHz}$ was used. The shadow fading standard deviation was set to $2 \mathrm{~dB}$. One hundred UE unitss were placed at random locations within each of four hotspot areas. Figure 


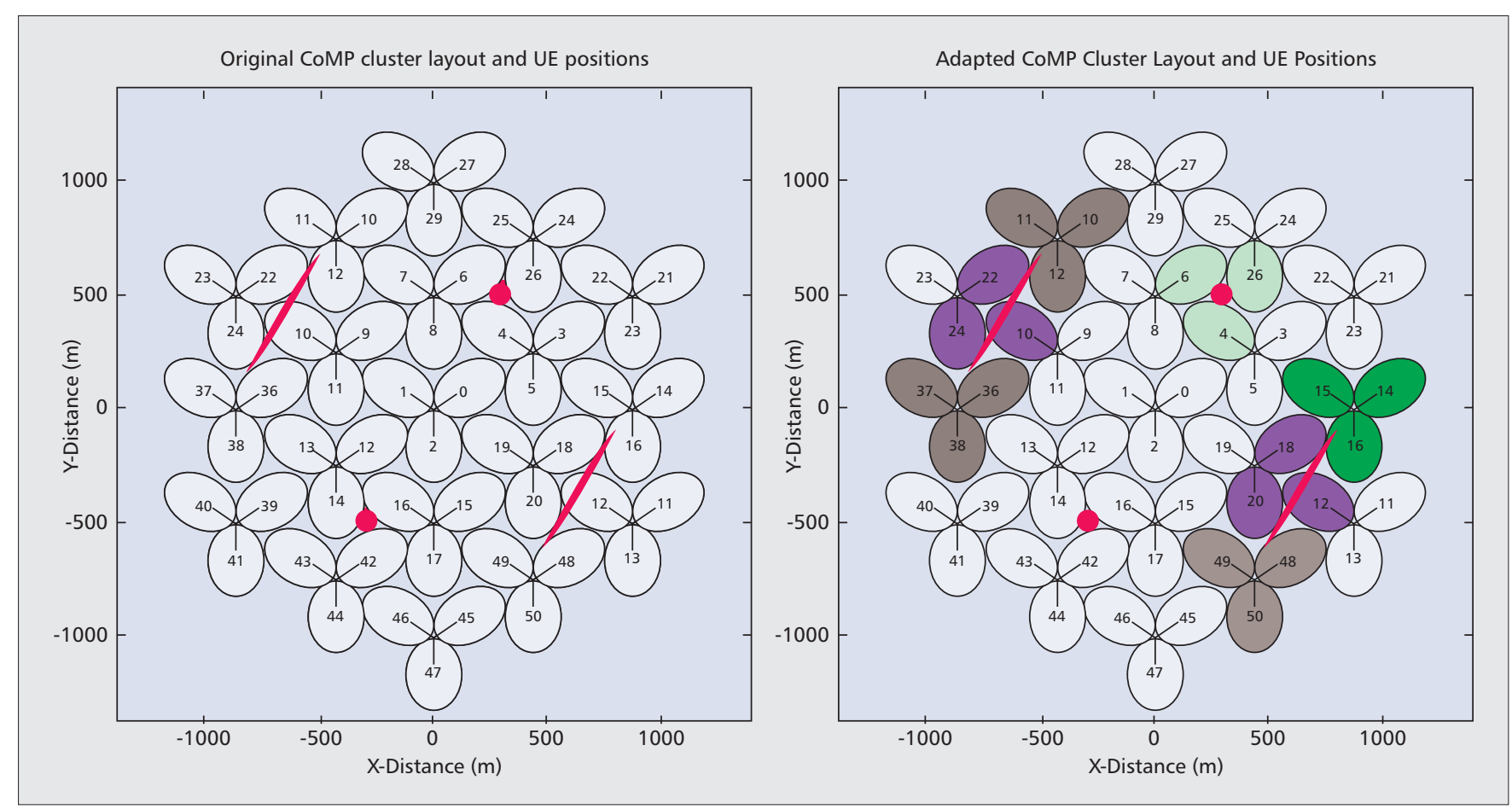

Figure 7. Cell layout with UE positions and selected clusters: a) no clustering; b) adaptive clustering.

$7 \mathrm{~b}$ shows the result of the designed clustering algorithm, which was configured to obtain the optimal solution for a disjoint set of clusters with up to three sectors. The colors represent the different clusters. The clustering algorithm took only long-term average received power measurements from UE into account in case they were higher than $-120 \mathrm{dBm}$. It is apparent from the figure that this concept managed to form clusters around the UE hotspots and avoided clusters in regions where not needed. The mean geometry gain due to adaptive clustering was about $6 \mathrm{~dB}$ for this scenario compared to LTE Release 8.

\section{BACKHAUL FOR COMP}

\section{ARCHITECTURE AND TECHNOLOGIES}

COMP approaches need to exchange direct information between cells, with different requirements of necessary backhaul throughput and latency. Intra-site COMP can be realized without any impact on backhaul. In the case of deployment of remote radio units connected to a centralized baseband processing unit via Ethernet or fiber links, COMP backhaul requirements should also be no obstacle.

For connectivity between sites, the logical X2 interface could be used. This could either be a direct physical link or a multihop link, depending on the network's backhaul architecture. The delay depends on the network topology, network node processing delay and line delay (usually speed of light). Gigabit Ethernet speeds of up 10 $\mathrm{Mb} / \mathrm{s}$ and delays of $0.1-20 \mu$ s with additional delays due to switching equipment. Other suitable candidates are conventional and millimeterwave microwave, with speeds up to of $800 \mathrm{Mb} / \mathrm{s}$ or $10 \mathrm{~Gb} / \mathrm{s}$, respectively, and delays as low as 150 $\mu \mathrm{s} / \mathrm{hop}$.

\section{LATENCY REQUIREMENTS}

COMP has to be integrated with the hybrid automatic repeat request (HARQ) process; thus, the backhaul latency will put some limits on this, suggesting a maximum latency of $1 \mathrm{~ms}$ without LTE standard modification.

Another impact of backhaul latency is that the exchanged channel information is outdated. For example, a minor performance degradation was estimated for coordinated scheduling considering a X2 latency of $6 \mathrm{~ms}$. In [15] a DL COMP capacity gain reduction of 20 percent is estimated for joint transmission with 5 ms backhaul latency at $3 \mathrm{~km} / \mathrm{h}$.

\section{CAPACITY REQUIREMENTS}

COMP schemes require the exchange of channel state information, control data, user data, and received signals, in a preprocessed or quantized format.

As shown earlier and in [16, 17], the backhaul requirements vary strongly from a few megabits per second up to $4 \mathrm{~Gb} / \mathrm{s}$ for different COMP approaches, considering a $10 \mathrm{MHz}$ LTE X2 link. This also depends on the cluster size. Earlier we showed an example of how backhaul can be reduced significantly even without major performance losses.

To conclude, state-of-the-art backhaul technology can support COMP in principle. However, the cost of additional backhaul and access capacity gains has to be balanced in a network deployment.

\section{CONCLUSIONS AND OUTLOOK}

This article has shown that coordination of cells in wide-area systems is not only beneficial for average spectral efficiency and cell edge data 
COMP schemes for

the uplink range

from joint multi-cell

scheduling to more

complex joint

detection, and can

be centralized or

decentralized. In the

downlink, the

schemes range from

less complex

coordinated

scheduling to more

challenging joint

processing

approaches. rates, but can also be implemented. COMP was demonstrated for uplink and downlink in two testbeds in urban areas. COMP schemes for the UL range from joint multicell scheduling to more complex joint detection, and can be centralized or decentralized. In the DL the schemes range from less complex coordinated scheduling to more challenging joint processing approaches.

From the technical as well as economic points of view, intrasite cooperation will be much easier to realize. However, intersite cooperation will be needed in order to exhaust the full interference reduction potential of base station cooperation. The combination of joint processing at one site with joint scheduling between the sites is of great interest as it provides promising gains with limited backhaul.

The following challenges needs to be addressed in order to benefit from the promising COMP gains:

- Backhaul with low latency and high bandwidth. Today's backhaul technologies can support COMP, but more effort is needed to reduce the amount of data exchanged between the sites.

- Clustering and multisite scheduling.

- Channel estimation and efficient feedback (for DL COMP).

- Synchronization between sites is feasible today, but the cell area where COMP can be applied may be limited by the length of the cyclic prefix.

- Combination of UL and DL COMP and their integration into the LTE standard.

This article, and the EASY-C project, have already given some answers on COMP. Ongoing efforts to address the challenges in the research community - such as the ARTIST4G project and 3GPP standardization - are important to gain more insight into achievable spectral efficiency gains and the complexity of different approaches.

\section{ACKNOWLEDGMENT}

The authors acknowledge the excellent cooperation of all project partners within the EASY-C project and the support of the German Federal Ministry of Education and Research (BMBF).

\section{REFERENCES}

[1] P. Marsch, S. Khattak, and G. Fettweis, "A Framework for Determining Realistic Capacity Bounds for Distributed Antenna Systems," Proc. IEEE Info. Theory Wksp. '06, Chengdu, China, Oct. 22-26, 2006.

[2] K. M. Karakayli, G. J. Foschini, and R. A. Valenzuela. "Network Coordination for Spectrally Efficient Communications in Cellular Systems," IEEE Wireless Commun., vol. 13, no. 4, Aug. 2006, pp. 56-61.

[3] P. Marsch and G. Fettweis, "On Multi-Cell Cooperative Transmission in Backhaul-Constrained Cellular Systems," Annales des Télécommun., vol. 63, no. 5-6, May 2008.

[4] V. Jungnickel et al., "Interference Aware Scheduling in the Multiuser MIMO-OFDM Downlink," IEEE Commun. Mag., vol. 47, no. 6, June 2009.

[5] V. Jungnickel et al., "Field Trials using Coordinated Multi-Point Transmission in the Downlink," 3rd IEEE Int'I. Wksp. Wireless Distrib. Net., IEEE PIMRC, Sept. 2010.

[6] P. Marsch, Coordinated Multi-Point under a Constrained Backhaul and Imperfect Channel Knowledge, Ph.D. thesis.

[7] A. Müller and P. Frank, "Cooperative Interference Prediction for Enhanced Link Adaptation in the 3GPP LTE Uplink," IEEE VTC-Spring, 2010.
[8] A. Müller and P. Frank, "Performance of the LTE Uplink with Intra-Site Joint Detection and Joint Link Adaptation," IEEE VTC-Spring, 2010.

[9] M. Grieger et al., "Field Trial Results for a Coordinated Multi-Point (CoMP) Uplink in Cellular Systems," Proc. ITG/IEEE Wksp. Smart Antennas '10, Bremen, Germany, Feb. 23-24, 2010

[10] V. Kotzsch and G. Fettweis. "Interference Analysis in Time and Frequency Asynchronous Network MIMO OFDM Systems," IEEE WCNC '10, Sydney, Australia, Apr. 18-21, 2010

[11] 3GPP TR 36.814, "Further Advancements for E-UTRA Physical Layer Aspects," Release 9, v. 9.0.0, Mar. 2010.

[12] J. Giese and M. A. Awais, "Performance Upper Bounds for Coordinated Beam Selection in LTE-Advanced," Proc. ITG/IEEE Wksp. Smart Antennas '10, Bremen, Germany, Feb. 23-24, 2010.

[13] G. Fettweis et al.," "Field Trial Results for LTE-Advanced Concepts," Proc. IEEE ICASSP '10, Dallas, TX, Mar. 14-19, 2010.

[14] L. Thiele, V. Jungnickel, and T. Haustein, "Interference Management for Future Cellular OFDMA Systems Using Coordinated Multi-Point Transmission "IEICE Trans. Commun., Special Issue on Wireless Distributed Networks, Dec. 2010.

[15] S. Brueck et al., "Centralized Scheduling for JointTransmission Coordinated Multi-Point in LTE-Advanced," Proc. ITG/IEEE Wksp. Smart Antennas '10, Bremen, Germany, Feb. 23-24, 2010.

[16] C. Hoymann, L. Falconetti, and R. Gupta, "Distributed Uplink Signal Processing of Cooperating Base Stations based on IQ Sample Exchange," Proc. IEEE ICC '09, Dresden, Germany, June 14-18, 2009.

[17] L. Falconetti, C. Hoymann, and R. Gupta, "Distributed Uplink Macro Diversity for Cooperating Base Stations," Proc. IEEE ICC '09, Dresden, Germany, June 14-18, 2009.

\section{AdDITIONAL READING}

[1] R. Irmer et al. "Multisite Field Trial for LTE and Advanced Concepts," IEEE Commun. Mag., vol. 47, no. 2, Feb. 2009, pp. 92-98.

\section{BIOGRAPHIES}

RALF IRMER [SM] (ralf.irmer@vodafone.com) received his Dipl-Ing. and Dr.-Ing. degrees from Technische Universität Dresden in 2000 and 2005, respectively. He joined Vodafone Group R\&D in 2005, where he leads the Wireless Access Group, which is responsible for evolution of LTE, WiFi, and other technologies, and defining Vodafone's future network architecture. Before, he worked for five years as a research associate at TU Dresden. He holds several patents, and has published more than 30 conference and journal publications. He had a leading role in severa research projects, including WIGWAM, WINNER, and EASYC. He is a member of VDE and IET.

HEINZ DRoste (Heinz.Droste@telekom.de) received his Dipl.Ing degree 1991 from the Open University, Hagen. Since then he has been working for Deutsche Telekom at a variety of mobile communication related R\&D projects. Antennas and radio wave propagation belong to his knowledge field as well as system-level simulation and radio network planning. More recently he extended his expertise to the field of techno-economical evaluations. In the framework of EASY-C he coordinates the partner activities in Working Group 1, "Algorithm and Concepts."

PATRICK MARSCH (marsch@ifn.et.tu-dresden.de) received his Dipl.-Ing. and Dr.-Ing. degrees from Technische Universität Dresden in 2004 and 2010, respectively, after completing an apprenticeship at Siemens AG and studying at the TU Dresden and McGill University, Montréal, Canada. After an internship with Philips Research East Asia in Shanghai, P.R. China, he joined the Vodafone Chair in 2005. He is the technical project coordinator of EASY-C, and is currently heading a research group on the analysis and optimization of cellular systems.

MICHAEL GRIEGER received his Dipl.-Ing. from DHBW Stuttgart in 2005 and his M.Sc. from the Technische Universität Dresden in March 2009. In 2008, funded by the Herbert Quandt/ALTANA Foundation, he studied at CTU, Prague. During his Master's thesis, he conducted research in Prof. John Cioffi's group at Stanford University on multicell sig- 
nal processing, which continues to be his major research focus today. An aspect of his research is the comparison of information theoretic results to those of the "real world" using field trials.

GeRHARD FETTWEIS [F] (fettweis@ifn.et.tu-dresden.de) earned his Dipl.-Ing. (1986) and Ph.D. (1990) degrees from Aachen University of Technology (RWTH), Germany. From 1990 to 1991 he was a visiting scientist at the IBM Almaden Research Center, San Jose, California, working on signal processing for disk drives. From 1991 to 1994 he was with TCSI Inc., Berkeley, California, responsible for signal processor development. Since 1994 he holds the Vodafone Chair at TU Dresden. He is coordinating the research project EASY-C.

HANS-Peter MAYER (Hans-Peter.Mayer@alcatel-lucent.de) received his Ph.D. degree in physics from the University of Tübingen in 1987. He joined Alcatel-Lucent and worked on high-speed optoelectronic and WDM components until 1995. From 1996 to 1999 , he has been responsible for early UMTS system studies, followed by the realization of first UMTS and HSPA trial systems. Within Bell Labs, he is currently responsible for the Advanced MAC department with a focus on projects related to LTE-Advanced.

STEFAN BRUECK (sbrueck@qualcomm.com) studied mathematics and electrical engineering at the University of Technology Darmstadt, Germany, and Trinity College Dublin, Ireland. He received his Dipl.-Math. and Dr.-Ing. degrees in
1994 and 1999, respectively. From 1999 to 2008 he was working for Lucent Technologies and Alcatel-Lucent in Bell Labs and UMTS Systems Engineering, where he was responsible for the MAC layer design of the HSPA base station. In May 2008 he joined Qualcomm Germany and currently leads the Radio Systems R\&D activities in the Corporate R\&D Centre Nuremberg. He is involved in several research projects on LTE-Advanced.

LARS THIELE [S'05] received his Dipl.-Ing. (M.S.) degree in electrical engineering from TU Berlin in 2005. Currently he is working towards his Dr.-Ing. (Ph.D.) degree at the Fraunhofer Heinrich Hertz Institute (HHI), Berlin. He has contributed to receiver and transmitter optimization under limited feedback, performance analysis for MIMO transmission in cellular ODFM systems, and fair resource allocation. He has authored and co-authored about 40 conference and journal papers in the area of mobile communications.

VOLKER JUNGNICKEL [M'99] (jungnickel@hhi.de) received a Dr rer. nat. (Ph.D.) degree in physics from Humboldt Universität zu Berlin in 1995. He worked on semiconductor quantum dots and laser medicine and joined $\mathrm{HHI}$ in 1997. He is a lecturer at TU Berlin and head of the cellular radio team at $\mathrm{HHI}$. He has contributed to high-speed indoor wireless infrared links, $1 \mathrm{~Gb} / \mathrm{s}$ MIMO-OFDM radio transmission, and initial field trials for LTE and LTE-Advanced. He has authored and co-authored more than 100 conference and journal papers on communications engineering. 